$02,05,11$

\title{
Влияние межузельного кулоновского взаимодействия на киральную сверхпроводимость при наличии неколлинеарного спинового упорядочения
}

\author{
(C) В.В. Вальков, А.О. Злотников
}

\author{
Институт физики им. Л.В. Киренского ФИЦ КНЦ СО РАН, \\ Красноярск, Россия \\ E-mail: vvv@iph.krasn.ru
}

\begin{abstract}
Исследовано влияние межузельного кулоновского взаимодействия в планарной системе с треугольной решеткой на структуру кирального параметра порядка $\Delta(\mathbf{p})$ в фазе сосуществования сверхпроводимости и неколлинеарного $120^{\circ}$ магнитного упорядочения. Установлено, что кулоновские корреляции в отмеченной фазе инициируют состояние, в котором квазиимпульсная зависимость $\Delta(\mathbf{p})$ представима в виде суперпозиции киральных инвариантов, соответствующих $d_{x^{2}-y^{2}}+i d_{x y}$ и $p_{x}+i p_{y}$-типам симметрии. Показано, что включение кулоновского взаимодействия смещает положение нодальных точек $\Delta(\mathbf{p})$, изменяя тем самым условия реализации квантового топологического перехода.
\end{abstract}

Работа выполнена при поддержке фонда РФФИ, правительства Красноярского края и Краевого фонда науки (проекты № 16-02-00073-a, 16-42-243057-р-мол-а) и Комплексной программы СО РАН ІІ.2П (проект 0358-2015-002). А.О. Злотников благодарит за поддержку в рамках стипендии президента РФ СП-1370.2015.5.

DOI: 10.21883/FTT.2017.11.45044.03k

\section{1. Введение}

Интерес к сверхпроводимости с киральным типом симметрии параметра порядка обусловлен формированием в таком состоянии топологически нетривиальной фазы и, как следствие, возникновением краевых состояний [1]. После открытия сверхпроводимости в соединении $\mathrm{Na}_{x} \mathrm{CoO}_{2}$ было высказано предположение о реализации киральной $d_{x^{2}-y^{2}}+i d_{x y}$ сверхпроводящей фазы в квазидвумерных системах с треугольной решеткой [2]. Для такой сверхпроводящей фазы возможно формирование квантового топологического перехода по концентрации $[3,4]$. В точке перехода спектр элементарных возбуждений становится бесщелевым. С этими обстоятельствами связано неослабевающее внимание к системам с киральной симметрией сверхпроводящего параметра порядка.

В последнее время было установлено, что дальний магнитный порядок, как и спин-орбитальное взаимодействие, может приводить к реализации краевых майорановских состояний в топологическом сверхпроводнике с синглетным спариванием [5,6]. Для треугольной решетки данный эффект был продемонстрирован в случае неколлинеарного магнитного порядка со „страйповой“ структурой в киральной $d_{x^{2}-y^{2}}+i d_{x y}$ сверхпроводящей фазе [5]. Дальнейшие исследования показали [7], что для ансамбля фермионов Хаббарда в рамках $t-J$-модели при „страйповой“ магнитной структуре киральная симметрия сверхпроводящего параметра порядка невозможна. В то же время для магнитной структуры, соответствующей неколлинеарному $120^{\circ}$ упорядочению, киральная сверхпроводимость по-прежнему может реализовываться. Этот результат говорит о том, что майора- новские краевые состояния следует искать в однородной фазе сосуществования киральной сверхпроводимости и $120^{\circ}$ спинового упорядочения. Впоследствии для такой фазы в рамках квадратичного гамильтониана были найдены условия реализации майорановских мод [8].

Отсутствие точных решений для модели Гейзенберга на треугольной решетке приводило к неопределенности в вопросе о реализации той или иной магнитной структуры. Долгое время считалось, что в отмеченной модели реализуется состояние квантовой спиновой жидкости [9]. Однако в настоящее время для большинства исследователей предпочтительным считается сценарий реализации $120^{\circ}$ дальнего магнитного упорядочения с тремя подрешетками. Данное состояние наиболее выгодно с классической точки зрения, если $J_{2}<J_{1} / 8$, где $J_{1}, J_{2}$ - параметры обмена между ближайшими и следующими за ближайшими соседями. Для модели Хаббарда на треугольной решетке в рамках среднеполевого приближения [10] и слейв-бозонного представления [11] были построены фазовые диаграммы, демонстрирующие существование различных состояний, в том числе неколлинеарных и некомпланарных, со спиновым и зарядовым упорядочением. В данных подходах основное состояние со $120^{\circ}$ спиновым упорядочением сохраняется при допировании вблизи половинного заполнения. Наиболее интересен случай электронного допирования при положительном значении интеграла перескока между ближайшими узлами, так как считается, что данный режим на качественном уровне может описать электронную структуру $\mathrm{Na}_{x} \mathrm{CoO}_{2}$. В работе [12] на основе вариационного метода Монте-Карло продемонстрирована реализация фазы сосуществования киральной сверхпроводимости и $120^{\circ}$ магнитного упорядочения вблизи $n=1.1$. При 
этом магнитная фаза оставалась устойчивой вплоть до концентрации $n=1.4$. Значение намагниченности в данном подходе при половинном заполнении незначительно уменьшено до значения $M \approx 0.4$. При этом из теории спиновых волн следует результат $M \approx 0.25$ [13].

Известно, что межузельное кулоновское отталкивание в $t-J$-модели подавляет сверхпроводимость $d$-типа. Поэтому часто для описания сверхпроводящей фазы учитываются дополнительные вклады в механизм куперовской неустойчивости [14]. В системах с сильным межузельным отталкиванием для первой координационной сферы сверхпроводящая фаза может реализоваться, если имеет место спаривательное взаимодействие во второй координационной сфере. Другая возможность сохранения сверхпроводящего состояния при сильном кулоновском отталкивании возникает в том случае, когда из-за сложной элементарной ячейки спаривательное и кулоновское взаимодействия обладают разными симметрийными свойствами [15]. Такая ситуация складывается, например, в купратных сверхпроводниках и проявляется при теоретическом описании в рамках спин-фермионной модели [15], получающейся из модели Эмери в режиме сильных корреляций.

В настоящей работе изучено влияние межузельных кулоновских корреляций на формирование фазы сосуществования киральной сверхпроводимости и $120^{\circ}$ магнитного порядка при учете обменного взаимодействия в пределах двух координационных сфер. Кроме обычного подавления сверхпроводящего состояния, межузельное отталкивание при неколлинеарном магнитном упорядочении приводит к нетривиальному эффекту, связанному с модификацией квазиимпульсной зависимости сверхпроводящего параметра порядка. В результате решение для параметра порядка $\Delta(\mathbf{p})$ определяется не только киральными инвариантами $d_{x^{2}-y^{2}}+i d_{x y}$-типа, как в случае фазы без магнитного упорядочения, но также в нем возникают инварианты, характеризующиеся $p_{x}+i p_{y}$-типом симметрии. Примешивание дополнительных инвариантов обусловлено индуцированием триплетных по спиновому моменту спариваний в магнитоупорядоченном состоянии [16,17]. Такие спаривания характеризуются нечетным $p$-типом классификации по номеру неприводимого представления (величине орбитального момента) в случае четности волновой функции по частоте. При этом за счет симметрии треугольной решетки новые компоненты обладают киральным $p_{x}+i p_{y}$-типом симметрии. Следует отметить, что триплетные спаривания обусловлены именно межузельным кулоновским взаимодействием в магнитоупорядоченном состоянии, так как обменное взаимодействие в $t-J$-модели не обладает триплетным каналом сверхпроводимости.

Индуцирование вкладов от триплетных спариваний изменяет положение нодальных точек сверхпроводящего параметра порядка внутри зоны Бриллюэна. Это приводит к изменению условий формирования бесщелевых возбуждений в фазе сосуществования. В результате отмеченные эффекты будут сказываться на описании топологических переходов в фазе сосуществования киральной сверхпроводимости и неколлинеарного магнетизма.

\section{2. Гамильтониан сильно коррелированных фермионов на треугольной решетке}

Изучение влияния межузельного кулоновского отталкивания на фазу сосуществования киральной сверхпроводимости и $120^{\circ}$ спинового упорядочения проведем в рамках $t-J_{1}-J_{2}-V$-модели для треугольной решетки, рассмотренной ранее в работах $[3,4]$ при описании куперовской неустойчивости с $d_{x^{2}-y^{2}}+i d_{x y}$-типом симметрии параметра порядка в интеркаллированных кобальтитах натрия. Важность учета спаривательного обменного взаимодействия во второй координационной сфере, как было показано в работе [3], обусловливается несколькими факторами. Во-первых, включение таких спариваний при рассмотрении сверхпроводимости $d_{x^{2}-y^{2}}+i d_{x y}$-типа симметрии приводит к возникновению нодальных точек в зоне Бриллюэна, для которых сверхпроводящий параметр порядка обращается в нуль. Данное обстоятельство позволило разрешить противоречие, связанное с тем, что теоретические расчеты для треугольной решетки предсказывали наиболее выгодным $d_{x^{2}-y^{2}}+i d_{x y}$-канал куперовской неустойчивости, но большинство экспериментов указывало на реализацию бесщелевого характера сверхпроводимости в кобальтитах натрия. Во-вторых, как известно, межузельное кулоновское отталкивание в системах с сильными корреляциями в значительной мере препятствует $d$-типу сверхпроводимости. Однако наибольший вклад определяется кулоновским взаимодействием между ближайшими соседями, так как корреляции между следующими за ближайшими соседями зачастую подавлены из-за эффектов экранировки. В этой связи предполагается, что при учете межузельных кулоновских корреляций в качестве доминирующего вклада в куперовскую неустойчивость может выступать спаривательное взаимодействие между следующими за ближайшими соседями.

Ограничимся рассмотрением верхней хаббардовской подзоны с подпространством, состоящем из однократно $|\sigma\rangle$ и двукратно $|2\rangle$ занятых электронных состояний, как это было предложено для качественного описания кобальтитов натрия. Гамильтониан $t-J_{1}-J_{2}-V$-модели в атомном представлении имеет вид

$$
\begin{aligned}
H= & \sum_{f \sigma}(\varepsilon-\mu) X_{f}^{\sigma \sigma}+\sum_{f}(2 \varepsilon+U-2 \mu) X_{f}^{22}+\sum_{f m \sigma} t_{f m} X_{f}^{2 \bar{\sigma}} X_{m}^{\bar{\sigma} 2} \\
& +\sum_{f m} J_{f m}\left(X_{f}^{\uparrow \downarrow} X_{m}^{\downarrow \uparrow}-X_{f}^{\uparrow \uparrow} X_{m}^{\downarrow \downarrow}\right)+\frac{V}{2} \sum_{f \delta} \hat{n}_{f} \hat{n}_{f+\delta},
\end{aligned}
$$

где $\varepsilon-$ затравочная энергия электрона, $\mu-$ химпотенциал, $U$ - величина внутриатомного кулоновского 
отталкивания, $t_{f m}-$ интенсивность перескоков электронов, $J_{f m}$ - параметр обменного взаимодействия. Операторы Хаббарда выражаются через одноузельные электронные состояния следующим образом: $X^{n m}=|n\rangle\langle m|$, где $n, m=\uparrow, \downarrow, 2$. Действие операторов Хаббарда на базис состояний определяется формулой $X^{n m}|p\rangle=\delta_{m p}|n\rangle$. Последнее слагаемое гамильтониана описывает кулоновское взаимодействие между электронами на ближайших узлах с параметром $V, \hat{n}_{f}=X_{f}^{\uparrow \uparrow}+X_{f}^{\downarrow \downarrow}+2 X_{f}^{22}-$ оператор числа электронов на узле.

\section{3. Функции Грина для фазы сосуществования сверхпроводимости и неколлинеарного спинового упорядочения}

Для решения поставленной задачи воспользуемся методом диаграммной техники для операторов Хаббарда [18] и определим функции Грина в мацубаровском представлении:

$$
D_{\alpha, \beta}\left(f \tau ; f^{\prime} \tau^{\prime}\right)=-\left\langle T_{\tau} \tilde{X}_{f}^{\alpha}(\tau) \tilde{X}_{f^{\prime}}^{-\beta}\left(\tau^{\prime}\right)\right\rangle,
$$

где $\alpha, \beta$ - индексы, используемые для обозначения пары одноузельных состояний. При неколлинеарном магнитном упорядочении со средним значением спина на узле $\left\langle\mathbf{S}_{f}\right\rangle=M\left(\cos \left(\mathbf{Q} \mathbf{R}_{f}\right),-\sin \left(\mathbf{Q} \mathbf{R}_{f}\right), 0\right)$ фурьепреобразование для функций Грина записывается в виде

$$
\begin{aligned}
D_{\alpha, \beta}\left(f \tau ; f^{\prime} \tau^{\prime}\right) & =\frac{T}{N} \sum_{\omega_{n}} \sum_{\mathbf{p}_{1} \mathbf{p}_{2}} \exp \left[-i \omega_{n}\left(\tau-\tau^{\prime}\right)\right. \\
& \left.+i \mathbf{p}_{1} \mathbf{R}_{f}-i \mathbf{p}_{2} \mathbf{R}_{f^{\prime}}\right] \tilde{D}_{\alpha, \beta}\left(\mathbf{p}_{1}, \mathbf{p}_{2} ; i \omega_{n}\right) .
\end{aligned}
$$

В результате непосредственного решения системы уравнений для функций Грина устанавливается связь между квазиимпульсами $\mathbf{p}_{1}, \mathbf{p}_{2}$

$$
\begin{aligned}
\tilde{D}_{\sigma 2, \sigma 2}\left(\mathbf{p}_{1}, \mathbf{p}_{2} ; i \omega_{n}\right)= & \delta\left(\mathbf{p}_{1}-\mathbf{p}_{2}\right) D_{\sigma 2, \sigma 2}\left(\mathbf{p}_{1}, i \omega_{n}\right), \\
\tilde{D}_{\sigma 2, \bar{\sigma} 2}\left(\mathbf{p}_{1}, \mathbf{p}_{2} ; i \omega_{n}\right)= & \delta\left(\mathbf{p}_{1}+\eta_{\sigma} \mathbf{Q}-\mathbf{p}_{2}\right) \\
& \times D_{\sigma 2, \bar{\sigma} 2}\left(\mathbf{p}_{1}, \mathbf{p}_{1}+\eta_{\sigma} \mathbf{Q} ; i \omega_{n}\right),
\end{aligned}
$$

Видно, что для функций Грина, в которых проекция спинового момента фермиона не изменяется, связь между квазиимпульсами определяется законом сохранения. Для функций Грина, описывающих процессы с переворотом спина, квазиимпульсы связаны вектором магнитной структуры $\mathbf{Q}$ для фермиона с $\sigma=\uparrow$ и $-\mathbf{Q}$ для фермиона с $\sigma=\downarrow$.

Для краткости матричную функцию Грина запишем в блочном виде

$$
\hat{D}=\left(\begin{array}{cc}
\hat{D}_{1 \uparrow}\left(\mathbf{p}, i \omega_{n}\right) & \hat{D}_{2 \uparrow}\left(\mathbf{p}, \mathbf{p}-\mathbf{Q} ; i \omega_{n}\right) \\
\hat{D}_{2 \downarrow}\left(\mathbf{p}-\mathbf{Q}, \mathbf{p} ; i \omega_{n}\right) & \hat{D}_{1 \downarrow}\left(\mathbf{p}-\mathbf{Q}, i \omega_{n}\right)
\end{array}\right),
$$

где

$$
\begin{aligned}
& \hat{D}_{1 \sigma}\left(\mathbf{p}, i \omega_{n}\right)=\left(\begin{array}{ll}
D_{\bar{\sigma} 2, \bar{\sigma} 2}\left(\mathbf{p}, i \omega_{n}\right) & D_{\bar{\sigma} 2,2 \sigma}\left(\mathbf{p}, i \omega_{n}\right) \\
D_{2 \sigma, \bar{\sigma} 2}\left(\mathbf{p}, i \omega_{n}\right) & D_{2 \sigma, 2 \sigma}\left(\mathbf{p}, i \omega_{n}\right)
\end{array}\right), \\
& \hat{D}_{2 \sigma}\left(\mathbf{p}_{1}, \mathbf{p}_{2} ; i \omega_{n}\right)= \\
& =\left(\begin{array}{ll}
D_{\bar{\sigma} 2, \sigma 2}\left(\mathbf{p}_{1}, \mathbf{p}_{2} ; i \omega_{n}\right) & D_{\bar{\sigma} 2,2 \hat{\sigma}}\left(\mathbf{p}_{1}, \mathbf{p}_{2} ; i \omega_{n}\right) \\
D_{2 \sigma, \sigma 2}\left(\mathbf{p}_{1}, \mathbf{p}_{2} ; i \omega_{n}\right) & D_{2 \sigma, 2 \bar{\sigma}}\left(\mathbf{p}_{1}, \mathbf{p}_{2} ; i \omega_{n}\right)
\end{array}\right) .
\end{aligned}
$$

Как известно, матричную функцию Грина можно представить в виде произведения: $\hat{D}=\hat{G} \cdot \hat{P}$, где $\hat{P}$ - матрица силового оператора, которую запишем в беспетлевом приближении:

$$
\hat{P}=\left(\begin{array}{cccc}
f_{2 \uparrow} & 0 & M & 0 \\
0 & f_{2 \downarrow} & 0 & M \\
M & 0 & f_{2 \downarrow} & 0 \\
0 & M & 0 & f_{2 \uparrow}
\end{array}\right)
$$

где $f_{2 \sigma}=\left\langle X_{f}^{\bar{\sigma} \bar{\sigma}}\right\rangle+\left\langle X_{f}^{22}\right\rangle$. Так как в рассматриваемой магнитной фазе $\left\langle S_{f}^{z}\right\rangle=0$, то $f_{2 \sigma}=n / 2$, где $n=\left\langle\hat{n}_{f}\right\rangle$. При переходе к представлению для силового оператора учитывалось, что при неколлинеарном спиновом упорядочении отличны от нуля средние значения от недиагональных операторов: $\left\langle X_{f}^{\uparrow \downarrow}\right\rangle=M \exp \left(-i \mathbf{Q R}_{f}\right)$, $\left\langle X_{f}^{\downarrow \uparrow}\right\rangle=M \exp \left(i \mathbf{Q R}_{f}\right)$.

Уравнение Дайсона-Горькова для функции $\hat{G}$ имеет стандартный вид

$$
\hat{G}=\hat{G}^{(0)}+\hat{G}^{(0)} \hat{\Sigma} \hat{G}
$$

где $\hat{G}^{(0)}$ - матричная функция Грина для неколлинеарной магнитной фазы (диаграммы для данных функций приведены в [7]). В массовом операторе $\hat{\Sigma}$ учитываются только аномальные компоненты, ответственные за куперовскую неустойчивость, в однопетлевом приближении

$$
\begin{aligned}
\Delta_{1}(\mathbf{p})= & \frac{T}{N} \sum_{\omega_{n} \mathbf{q}}\left[J_{\mathbf{p}-\mathbf{q}} G_{2 \downarrow, \uparrow 2}\left(\mathbf{q}, i \omega_{n}\right)\right. \\
& \left.-\left(J_{\mathbf{p}-\mathbf{q}}-V_{\mathbf{p}-\mathbf{q}}\right) G_{2 \uparrow, \downarrow 2}\left(\mathbf{q}, i \omega_{n}\right)\right], \\
\Delta_{2}(\mathbf{p})= & \frac{T}{N} \sum_{\omega_{n} \mathbf{q}}\left[J_{\mathbf{p}-\mathbf{q}} G_{2 \uparrow, \downarrow 2}\left(\mathbf{q}, i \omega_{n}\right)\right. \\
& \left.-\left(J_{\mathbf{p}-\mathbf{q}}-V_{\mathbf{p}-\mathbf{q}}\right) G_{2 \downarrow, \uparrow 2}\left(\mathbf{q}, i \omega_{n}\right)\right] .
\end{aligned}
$$

Из общих симметрийных соотношений для функций Грина следует, что $\Delta_{2}(\mathbf{p})=-\Delta_{1}(-\mathbf{p})$. В дальнейшем $\Delta_{1}(\mathbf{p}) \equiv \Delta(\mathbf{p})$. С учетом этого решение уравнения (8) представляется в виде

$\hat{G}^{-1}=\left(\begin{array}{cccc}i \omega_{n}-\xi_{\mathbf{p}} & -\Delta^{*}(\mathbf{p}) & -R_{\mathbf{p}-\mathbf{Q}} & 0 \\ -\Delta(\mathbf{p}) & i \omega_{n}+\xi_{\mathbf{p}} & 0 & R_{\mathbf{p}-\mathbf{Q}} \\ -R_{\mathbf{p}} & 0 & i \omega_{n}-\xi_{\mathbf{p}-\mathbf{Q}} & \Delta^{*}(-\mathbf{p}+\mathbf{Q}) \\ 0 & R_{\mathbf{p}} & \Delta(-\mathbf{p}+\mathbf{Q}) & i \omega_{n}+\xi_{\mathbf{p}-\mathbf{Q}}\end{array}\right)$,

где $\xi_{\mathbf{p}}=\varepsilon+U-\mu+J_{0}(1-n / 2)+V_{0} n+n t_{\mathbf{p}} / 2, J_{0}=6 J_{1}$ $+6 J_{2}, V_{0}=6 \mathrm{~V}, t_{\mathrm{p}}-$ фурье-образ интеграла переско- 
ка. Введены параметры, определяющие самосогласованное поле $R_{\mathbf{p}}=M\left(t_{\mathbf{p}}-J_{\mathbf{Q}}\right), \quad R_{\mathbf{p}-\mathbf{Q}}=M\left(t_{\mathbf{p}-\mathbf{Q}}-J_{\mathbf{Q}}\right)$, где $J_{\mathbf{Q}}=-3 J_{1}+6 J_{2}$ - значение фурье-образа обменного интеграла для волнового вектора $\mathbf{Q}=2 \pi / a(1 / \sqrt{3}, 1 / 3)$ $120^{\circ}$ магнитной структуры. Появление слагаемых, пропорциональных интегралам перескока, в эффективном обменном поле обусловлено учетом кинематического взаимодействия в неколлинеарной магнитной фазе [7].

\section{4. Спектр возбуждений и киральный параметр порядка фазы сосуществования}

Спектр элементарных возбуждений в фазе сосуществования сверхпроводимости и неколлинеарного магнитного порядка определяется из выражения

$$
E_{\lambda \mathbf{p}}=\sqrt{\begin{array}{c}
\frac{1}{2}\left(\xi_{\mathbf{p}}^{2}+\xi_{\mathbf{p}-\mathbf{Q}}^{2}+|\Delta(\mathbf{p})|^{2}+|\Delta(-\mathbf{p}+\mathbf{Q})|^{2}\right)+ \\
+R_{\mathbf{p}} R_{\mathbf{p}-\mathbf{Q}}+(-1)^{\lambda} v_{\mathbf{p}}^{2}
\end{array}},
$$

где

$$
\begin{aligned}
v_{\mathbf{p}}^{2} & =\left\{\frac{1}{4}\left(\xi_{\mathbf{p}}^{2}-\xi_{\mathbf{p}-\mathbf{Q}}^{2}+|\Delta(\mathbf{p})|^{2}-|\Delta(-\mathbf{p}+\mathbf{Q})|^{2}\right)^{2}\right. \\
& \left.+R_{\mathbf{p}} R_{\mathbf{p}-\mathbf{Q}}\left[\left(\xi_{\mathbf{p}}+\xi_{\mathbf{p}-\mathbf{Q}}\right)^{2}+|\Delta(\mathbf{p})+\Delta(-\mathbf{p}+\mathbf{Q})|^{2}\right]\right\}^{1 / 2} .
\end{aligned}
$$

Следует отметить, что данный спектр обладает свойством $E_{\lambda \mathbf{p}}=E_{\lambda,-\mathbf{p}+\mathbf{Q}}$.

Аналитические выражения для функций Грина определяются из обратной матрицы (11). Необходимо отметить, что для нахождения аномальной функции $G_{2 \downarrow, \uparrow 2}\left(\mathbf{p}, i \omega_{n}\right)$, входящей в выражение для сверхпроводящего параметра порядка (9), можно воспользоваться полученными выше формулами, если в (11) сделать замену $\mathbf{p} \rightarrow \mathbf{p}+\mathbf{Q}$. Проводя суммирование по мацубаровским частотам в (9), получаем интегральное уравнение самосогласования, решения которого определяют квазиимпульсную зависимость сверхпроводящего параметра порядка:

$$
\Delta(\mathbf{p})=\frac{1}{N} \sum_{\mathbf{q}} \Phi(\mathbf{p}, \mathbf{q}) \Delta(\mathbf{q}),
$$

где ядро уравнения определяется выражением

$$
\begin{aligned}
& \Phi(\mathbf{p}, \mathbf{q})=\sum_{\lambda=1}^{2}\left\{\left[( J _ { \mathbf { p } + \mathbf { q } } + J _ { \mathbf { p } - \mathbf { q } } - V _ { \mathbf { p } - \mathbf { q } } ) \left(E_{\lambda \mathbf{q}}^{2}-\xi_{\mathbf{q}-\mathbf{Q}}^{2}\right.\right.\right. \\
& \left.\left.-|\Delta(-\mathbf{q}+\mathbf{Q})|^{2}\right)+\left(J_{\mathbf{p}+\mathbf{q}-\mathbf{Q}}+J_{\mathbf{p}-\mathbf{q}+\mathbf{Q}}-V_{\mathbf{p}+\mathbf{q}-\mathbf{Q}}\right) R_{\mathbf{q}} R_{\mathbf{q}-\mathbf{Q}}\right] \\
& \left.\times \frac{(-1)^{\lambda} \tanh \left(E_{\lambda \mathbf{q}} / 2 T\right)}{2 E_{\lambda \mathbf{q}}\left(E_{2 \mathbf{q}}^{2}-E_{1 \mathbf{q}}^{2}\right)}\right\} .
\end{aligned}
$$

Ранее в работе [7] в линеаризованном по параметpy $\Delta(\mathbf{p})$ приближении, справедливому вблизи критической температуры, было показано, что решение уравнения (14) при наличии $120^{\circ}$ магнитного упорядочения соответствует линейной суперпозиции инвариантов $d_{x^{2}-y^{2}}+i d_{x y}-$ и $p_{x}+i p_{y}$-типов

$$
\Delta(\mathbf{p})=2 \Delta_{21} \phi_{21}(\mathbf{p})+2 \Delta_{22} \phi_{22}(\mathbf{p})+2 \Delta_{11} \phi_{11}(\mathbf{p}),
$$

где киральные инварианты имеют вид:

$$
\begin{aligned}
& \phi_{21}(\mathbf{p})= \cos \left(p_{y}\right)-\cos \left(\sqrt{3} p_{x} / 2\right) \cos \left(p_{y} / 2\right) \\
&+i \sqrt{3} \sin \left(\sqrt{3} p_{x} / 2\right) \sin \left(p_{y} / 2\right), \\
& \phi_{22}(\mathbf{p})=\cos \left(\sqrt{3} p_{x}\right)-\cos \left(\sqrt{3} p_{x} / 2\right) \cos \left(3 p_{y} / 2\right) \\
&-i \sqrt{3} \sin \left(\sqrt{3} p_{x} / 2\right) \sin \left(3 p_{y} / 2\right), \\
& \phi_{11}(\mathbf{p})= \sin \left(p_{y}\right)+\cos \left(\sqrt{3} p_{x} / 2\right) \sin \left(p_{y} / 2\right) \\
&+i \sqrt{3} \sin \left(\sqrt{3} p_{x} / 2\right) \cos \left(p_{y} / 2\right) .
\end{aligned}
$$

Функции $\phi_{21}(\mathbf{p})$ и $\phi_{22}(\mathbf{p})$ определяют киральные инварианты $d_{x^{2}-y^{2}}+i d_{x y}$-типа, отвечающие спаривательным взаимодействиям между ближайшими и следующими за ближайшими соседями соответственно, а $\phi_{11}(\mathbf{p})-$ киральный инвариант $p_{x}+i p_{y}$-типа для первой координационной сферы.

За счет расщепленности ядра $\Phi(\mathbf{p}, \mathbf{q})$ интегральное уравнение (14) сводится к системе трех нелинейных алгебраических уравнений, позволяющих определить температурные зависимости аномальных амплитуд сверхпроводящего параметра порядка $\Delta(\mathbf{p})$ :

$$
\begin{gathered}
\left(1-a_{21}\right) \Delta_{21}-a_{22} \Delta_{22}-a_{11} \Delta_{11}=0 \\
-b_{21} \Delta_{21}+\left(1-b_{22}\right) \Delta_{22}-b_{11} \Delta_{11}=0 \\
c_{21} \Delta_{21}+c_{22} \Delta_{22}+\left(1+c_{11}\right) \Delta_{11}=0
\end{gathered}
$$

Коэффициенты этой системы уравнений зависят от аномальных амплитуд и определяются следующим образом:

$$
\begin{aligned}
a_{i j}= & \left(J_{1}-\frac{V}{2}\right) \frac{1}{N} \sum_{\mathbf{q} \lambda} \frac{(-1)^{\lambda} \phi_{i j}(\mathbf{q}) \tanh \left(E_{\lambda \mathbf{q}} / 2 T\right)}{E_{\lambda \mathbf{q}} \nu_{\mathbf{q}}^{2}} \\
& \times\left\{\cos \left(q_{y}\right)\left[E_{\lambda \mathbf{q}}^{2}-\xi_{\mathbf{q}-\mathbf{Q}}^{2}-|\Delta(-\mathbf{q}+\mathbf{Q})|^{2}\right]\right. \\
& \left.+\cos \left(q_{y}-Q_{y}\right) R_{\mathbf{q}} R_{\mathbf{q}-\mathbf{Q}}\right\}, \\
b_{i j}= & J_{2} \frac{1}{N} \sum_{\mathbf{q} \lambda} \frac{(-1)^{\lambda} \phi_{i j}(\mathbf{q}) \tanh \left(E_{\lambda \mathbf{q}} / 2 T\right)}{E_{\lambda \mathbf{q}} \nu_{\mathbf{q}}^{2}} \\
& \times\left\{\cos \left(\sqrt{3} q_{x}\right)\left[E_{\lambda \mathbf{q}}^{2}-\xi_{\mathbf{q}-\mathbf{Q}}^{2}-|\Delta(-\mathbf{q}+\mathbf{Q})|^{2}\right]\right. \\
& \left.+\cos \left(\sqrt{3}\left(q_{x}-Q_{x}\right)\right) R_{\mathbf{q}} R_{\mathbf{q}-\mathbf{Q}}\right\},
\end{aligned}
$$




$$
\begin{aligned}
c_{i j}= & \frac{V}{2} \frac{1}{N} \sum_{\mathbf{q} \lambda} \frac{(-1)^{\lambda} \phi_{i j}(\mathbf{q}) \tanh \left(E_{\lambda \mathbf{q}} / 2 T\right)}{E_{\lambda \mathbf{q}} v_{\mathbf{q}}^{2}} \\
& \times\left\{\sin \left(q_{y}\right)\left[E_{\lambda \mathbf{q}}^{2}-\xi_{\mathbf{q}-\mathbf{Q}}^{2}-|\Delta(-\mathbf{q}+\mathbf{Q})|^{2}\right]\right. \\
& \left.-\sin \left(q_{y}-Q_{y}\right) R_{\mathbf{q}} R_{\mathbf{q}-\mathbf{Q}\}}\right\}
\end{aligned}
$$

\section{5. Результаты численных расчетов}

На рис. 1 продемонстрированы концентрационные зависимости температуры возникновения сверхпроводимости с киральной симметрией параметра порядка при наличии $120^{\circ}$ магнитного упорядочения для различных значений параметра межузельного кулоновского взаимодействия $V$. Предполагается, что температура возникновения магнитного порядка намного выше критической температуры сверхпроводимости. Для намагниченности используется значение $M=1-n / 2$. Параметры $J_{1}=0.5 t_{1}, J_{2}=0.06 t_{1}$ - обменные константы между ближайшими и следующими за ближайшими соседями, $t_{1}$ - параметр перескока между ближайшими соседями $\left(t_{1} \sim 0.1 \mathrm{eV}\right)$. Для упрощения анализа эффекты дальних перескоков не учитываются. Видно, что увеличение параметра межузельного отталкивания приводит к ожидаемому подавлению сверхпроводимости. При значении $V=2 J_{1}$ и выше этого значения куперовская неустойчивость индуцируется за счет спаривательного взаимодействия во второй координационной сфере. Так как $J_{2} \ll J_{1}$, то при учете межузельных кулоновских корреляций переход в фазу сосуществования реализуется при довольно низких температурах (порядка нескольких К).

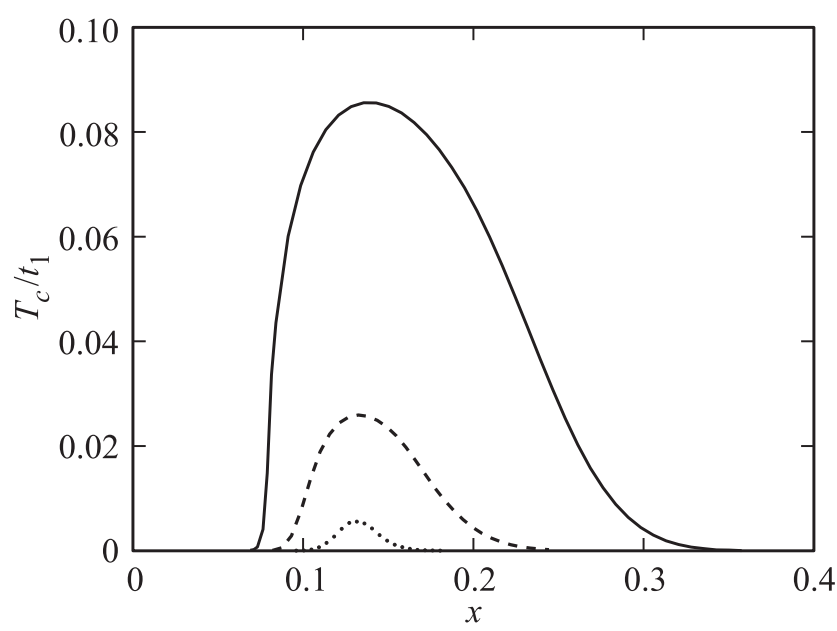

Рис. 1. Зависимость температуры перехода в фазу сосуществования киральной сверхпроводимости и $120^{\circ}$ спинового упорядочения от концентрации $n=1+x$ для различных значений параметра $V$ межузельного кулоновского отталкивания: $V=0.5 t_{1}$ (сплошная линия), $V=0.8 t_{1}$ (штриховая линия), $V=2 J_{1}=t_{1}$ (пунктирная линия).

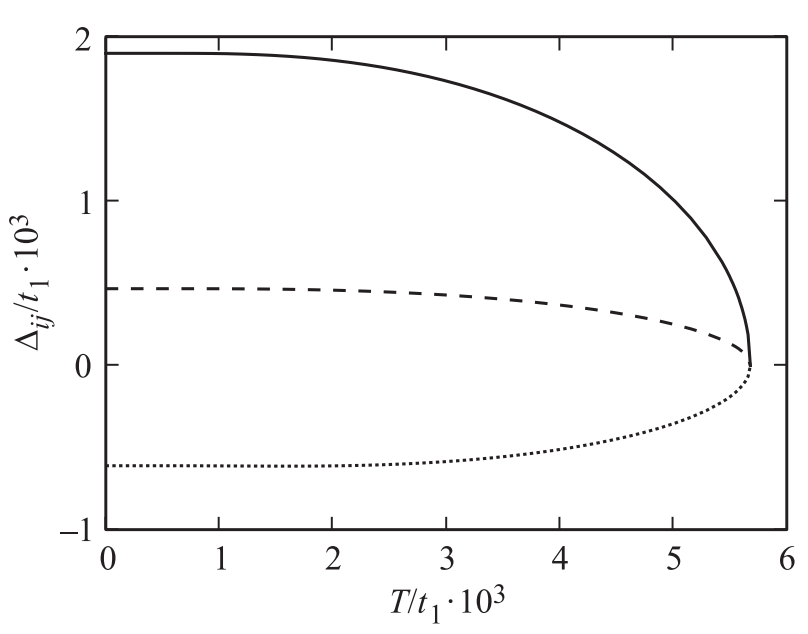

Рис. 2. Температурные зависимости амплитуд $\Delta_{22}$ (сплошная линия), $\Delta_{21}$ (штриховая линия), $\Delta_{11}$ (пунктирная линия) кирального сверхпроводящего параметра порядка для параметров $V=0.96 t_{1}, n=1.12$.

Как было показано выше, влияние межузельных кулоновских корреляций в фазе сосуществования сверхпроводимости и $120^{\circ}$ спинового упорядочения сводится не только к перенормировке аномальной амплитуды $\Delta_{21}$, но проявляется также в том, что решение для сверхпроводящего параметра порядка определяется суперпозицией киральных инвариантов $d_{x^{2}-y^{2}}+i d_{x y^{-}}$и $p_{x}+i p_{y}$-типов. Интенсивность примешивания кирального инварианта $p_{x}+i p_{y}$-типа определяется амплитудой $\Delta_{11}$. Ранее уже обсуждалось возникновение триплетных спариваний в затравочной синглетной сверхпроводящей фазе при наличии антиферромагнитного упорядочения [16,17]. Такие спаривания определяются как динамически индуцируемые за счет магнитного порядка. Это означает, что они развиваются отдельно от спариваний в триплетном канале сверхпроводимости, которые могут существовать и без магнитного упорядочения. Последние процессы не учитываются в рассматриваемой модели.

На рис. 2 представлены температурные зависимости аномальных амплитуд кирального сверхпроводящего параметра порядка для параметров $V=0.96 t_{1}, n=1.12$. Концентрация выбрана вблизи оптимального допирования. Видно, что для приведенного значения параметра кулоновского отталкивания вклад от спаривательного взаимодействия в первой координационной сфере, задаваемый амплитудой $\Delta_{21}$, существенно подавлен по сравнению с вкладом $\Delta_{22}$, определяющим спаривательное взаимодействие между следующими за ближайшими соседями. Амплитуда $\Delta_{11}$, характеризующая индуцируемые триплетные спаривания, принимает отрицательные значения. Это означает, что данные процессы не вносят дополнительного вклада в формировние куперовской неустойчивости на фоне дальнего магнитного порядка, а открывают дополнительный канал подавления сверхпроводимости. Однако данный эффект приводит только 


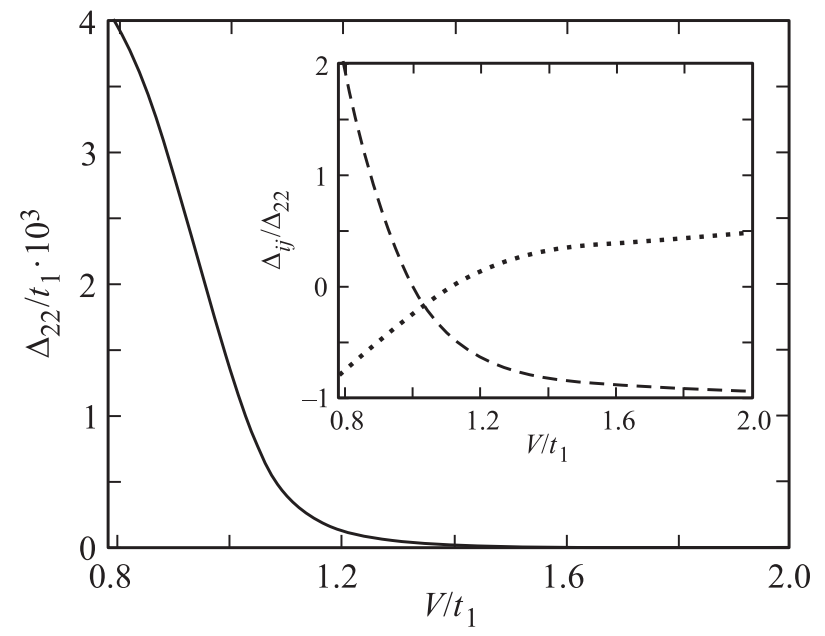

Рис. 3. Зависимость аномальной амплитуды $\Delta_{22}$ от параметра $V$ межузельного кулоновского отталкивания в пределе нулевой температуры для концентрации $n=1.12$. На вставке представлены зависимости амплитуд $\Delta_{21}, \Delta_{11}$ от $V$ по отношению к величине $\Delta_{22}$.

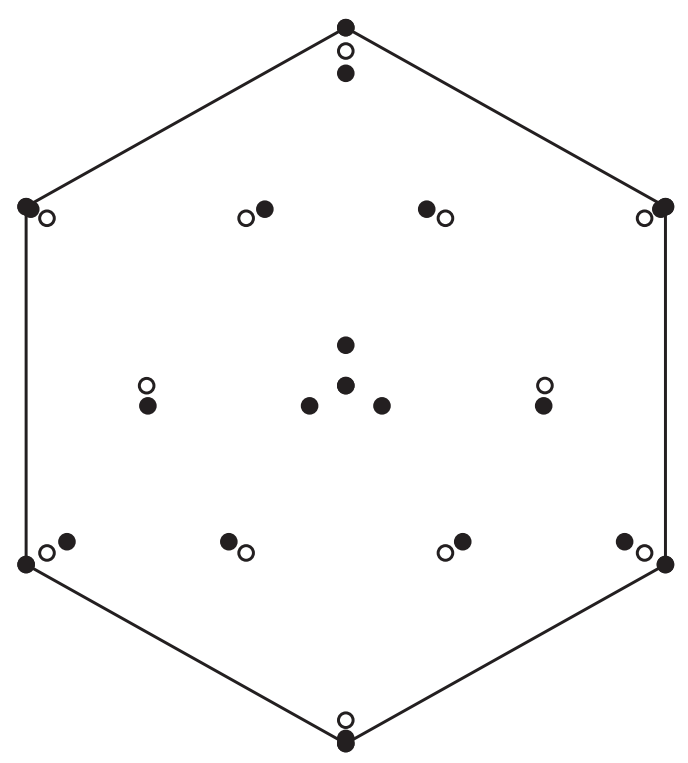

Рис. 4. Нодальные точки сверхпроводящего параметра порядка $\Delta(\mathbf{p})$ при учете кирального инварианта $p_{x}+i p_{y}$-типа (темные точки) и без его учета (светлые точки).

к слабому уменьшению температуры возникновения сверхпроводимости, так как величина $\Delta_{11}$ существенно меньше амплитуды $\Delta_{22}$.

На рис. 3 продемонстрировано уменьшение амплитуды $\Delta_{22}$ с ростом параметра $V$. Видно, что кулоновское отталкивание между ближайшими соседями не приводит к резкому подавлению сверхпроводимости, так как основной вклад в механизм куперовской неустойчивости при больших $V$ вносится спаривательным взаимодействием между следующими за ближайшими соседями. Рост величины кулоновского отталкивания обусловли- вает быстрое уменьшение аномальной амплитуды $\Delta_{21}$. При $V=2 J_{1}$ эта амплитуда обращается в нуль. При дальнейшем увеличении $V$ амплитуда $\Delta_{21}$ становится отрицательной. Данное поведение продемонстрировано на вставке к рис. 3 , на которой зависимости амплитуд $\Delta_{21}$ и $\Delta_{11}$ от параметра межузельного отталкивания приведены по отношению к величине $\Delta_{22}$. Интересно, что амплитуда $\Delta_{11}$ также испытывает смену знака и становится положительной при увеличении $V$, когда $\Delta_{21}<0$. Однако это происходит при очень малых критических температурах сверхпроводимости (десятые доли К).

Учет межузельного кулоновского отталкивания модифицирует квазиимпульсную зависимость сверхпроводящего параметра порядка за счет примешивания триплетного инварианта. Это приводит к изменению положения нодальных точек в зоне Бриллюэна, в которых сверхпроводящий параметр порядка обращается в нуль. На рис. 4 светлые кружки обозначают нодальные точки $\Delta(\mathbf{p})$, если $\Delta_{11}=0$, темные кружки получены при учете вкладов от триплетных спариваний. Значения для амплитуд $\Delta_{21}, \Delta_{22}$ выбраны при концентрации $n=1.12$ вблизи нулевой температуры (см. рис. 2). При $\Delta_{11}=0$ сверхпроводящий параметр порядка содержит нодальные точки в центре и на пересечении границ гексагональной зоны Бриллюэна, а также 12 точек внутри зоны: шесть из них расположены вблизи границ, что обусловлено малостью амплитуды $\Delta_{21}$, а оставшиеся шесть точек незначительно смещены относительно нодальных точек инварианта $\phi_{22}(p)$. Видно, что при учете триплетного инварианта с $\Delta_{11}=-0.32 \Delta_{22}$ нодальные точки, расположенные внутри зоны Бриллюэна, смещаются, а также возникают три новых точки вблизи $(0,0)$.

\section{6. Заключение}

Известно, что межузельное кулоновское взаимодействие обычно приводит к подавлению сверхпроводимости $d$-типа из-за ренормировки эффективного параметра спаривательного взаимодействия. Для рассмотренной нами фазы сосуществования киральной сверхпроводимости и неколлинеарного $120^{\circ}$ спинового упорядочения на треугольной решетке возникает дополнительный эффект. Он связан с тем, что кулоновское взаимодействие инициирует возникновение нетривиальной структуры сверхпроводящего параметра порядка, а именно $\Delta(\mathbf{p})$ представляется в виде линейной суперпозиции киральных инвариантов $d_{x^{2}-y^{2}}+i d_{x y}$ и $p_{x}+i p_{y}$-типов. Примешивание триплетного инварианта обусловлено только кулоновским взаимодействием и только для фазы сосуществования. При этом аномальная амплитуда, определяющая интенсивность вклада от триплетного спаривания, в широкой области параметров характеризуется отрицательными значениями. Это свидетельствует о возникновении дополнительного вклада, приводящего к подавлению фазы сосуществования сверхпроводимости и неколлинеарного магнитного упорядочения. 
В рассмотренном случае этот вклад не влияет существенно на критическую температуру перехода в фазу сосуществования, тем не менее полученный результат меняет качественную картину симметрийной классификации сверхпроводящего параметра порядка и имеет важное значение при исследовании топологических свойств сверхпроводящего состояния при наличии $120^{\circ}$ магнитного упорядочения.

\section{Список литературы}

[1] G.E. Volovik. Письма в ЖЭТФ 66, 492 (1997).

[2] G. Baskaran. Phys. Rev. Lett. 91, 097003 (2003).

[3] S. Zhou, Z. Wang. Phys. Rev. Lett. 100, 217002 (2008).

[4] В. В. Вальков, Т.А. Валькова, В.А. Мицкан. Письма в ЖЭТФ 100, 399 (2015).

[5] Y.-M. Lu, Z. Wang. Phys. Rev. Lett. 110, 096403 (2013).

[6] A.M. Black-Schaffer, K. Le Hur. Phys. Rev. B 92, 140503 (2015).

[7] В.В. Вальков, А.О. Злотников. Письма в ЖЭТФ 104, 512 (2016).

[8] V.V. Val'kov, A.O. Zlotnikov, A.D. Fedoseev, M.S. Shustin. J. Magn. Magn. Mater. DOI: j.jmmm.2016.12.093 (2017).

[9] P. W. Anderson. Mater. Res. Bull. 8, 153 (1973).

[10] K. Pasrija, S. Kumar. Phys. Rev. B 93, 195110 (2016).

[11] K. Jiang, S. Zhou, Z. Wang. Phys. Rev. B 90, 165135 (2014).

[12] C. Weber, A. Läuchli, F. Mila, T. Giamarchi. Phys. Rev. B 73, 014519 (2006).

[13] A.V. Chubukov, S. Sachdev, T. Senthil. J. Phys.: Condens. Matter 6, 8891 (1994).

[14] N.M. Plakida, V.S. Oudovenko. ЖЭТФ 146, 631 (2014).

[15] В.В. Вальков, Д.М. Дзебисашвили, М.М. Коровушкин, А.Ф. Барабанов. Письма в ЖЭТФ 103, 433 (2016).

[16] G.C. Psaltakis, E.W. Fenton. J. Phys. C 16, 3913 (1983).

[17] B. Kyung. Phys. Rev. B 62, 9083 (2000).

[18] Р.О. Зайцев. Диаграммные методы в теории сверхпроводимости и ферромагнетизма. Едиториал УРСС, М. (2004). $175 \mathrm{c}$. 